\section{Detection of Circularly Polarized $\gamma$-Rays and the Production of Polarized Electrons}

IN a recent communication ${ }^{1}$, it was pointed out that the $(n-\gamma)$ processes induced by polarized neutrons will in general lead to partially or totally polarized end-products. In particular, it was shown that for spin values of the initial and resulting nucleus of 0 and $\frac{1}{2}$, respectively, the $\gamma$-ray emitted in the direction of the spin of the incident neutron is completely circularly polarized. The same result holds true for the pair of spin values $\frac{1}{2}, 0$.

I wish to report now on some simple results concerning possible experiments with circularly polarized $\gamma$-rays. Choosing a special case of the Compton effect in which the quantum is scattered backwards and the electron forward in the direction of incidence, one finds that for moderately large quanta $\left(\frac{h \nu}{m c^{2}}>3\right)$ and an unpolarized electron initially at rest, the scattered electron is almost totally polarized, its spin pointing in the direction of propagation.

Similarly, if the electron is initially suitably polarized, the scattering cross-section will vary (in the approximation used) between zero and twice the value obtained for an unpolarized electron.

A detailed discussion, including that of other interactions between $\gamma$-rays and electrons, will be published in the Physical Review.

University of Southern California, OTto HALPERN Los Angeles 7. Aug. 16.

${ }^{1}$ Phys. Rev., 82, 753 (1951).

\section{The Helix as a Linear Accelerator for Protons}

AN electrostatic generator, designed for $4 \mathrm{MV}$., has been built at this Laboratory to accelerate positive ions, and the possibility of increasing the energy of protons from this machine to about $20 \mathrm{MeV}$. by means of a linear accelerator has been considered.

The disk-loaded wave-guide type of linear accelerator used for electron acceleration is impracticable for protons in this energy-range on account of the low phase-velocities required $(0 \cdot 1-0 \cdot 2 c)$. 'The highfrequency losses would be prohibitive and the tolerances in dimensions impossible to meet.

A more practicable form of proton accelerator is that suggested by W. Walkinshaw ${ }^{1}$, consisting of a circular wave-guide made up of a closely wound helical wire. Roughly, the travelling wave tends to follow the wire and is slowed down in the ratio of the pitch to the circumference of the helix. More accurately, the phase-velocity can be calculated from an approximate field solution given by Pierce and Schelkunoff ${ }^{2}$.

In a proposed design, assuming the helix radius to be $1 \mathrm{~cm}$., the optimum free-space wave-length is found to be 1 metre. If the radio-frequency power flowing down the guide is $2 \mathrm{MW}$., an accelerator 11 metres long will accelerate the protons from 4 to $20 \mathrm{MeV}$. The power dissipated in the helix is $380 \mathrm{~kW}$. ; the remainder can be fed back from output to input through an external line and suitable phasecorrecting network.

The chief difficulty in principle arises from the defocusing effect of the wave on the proton beam.

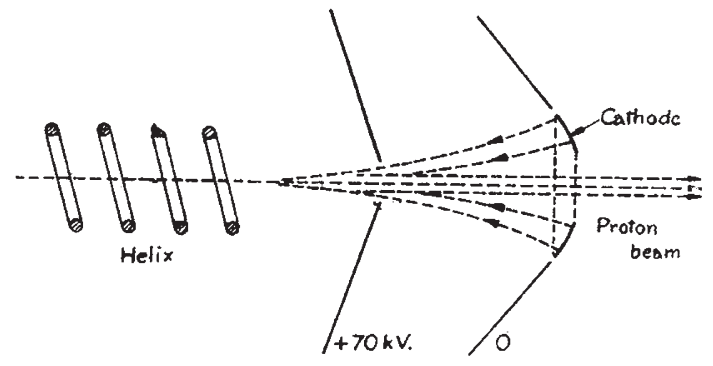

Possible arrangement of electronic gun

Those protons which get caught up by the wave and accelerated with it oscillate slowly about a stable phase-point which lies in front of the peak of the axial field; that is, in the region where there is a radial component of electric field outward. This radial component would cause a proton beam of initial radius $1 \mathrm{~mm}$. to spread and touch the helix in a distance of about 1 metre.

It is suggested that this may be prevented by sending a beam of electrons along the axis of the guide, in the opposite direction to the protons for convenience. The beam would be launched by some form of annular gun through which the proton beam could pass. The space charge per unit length of the electron beam must be sufficient to create a radial electrostatic field inward, equal to the maximum outward field arising from the wave. The energy of the beam must be such that no electrons are turned back by the wave at any point along the guide or at any phase of entry. Simple calculations indicate that a beam of 1 amp. at about $70 \mathrm{kV}$. is required. The electron gun may be pulsed in synchronism with the radio-frequency oscillator in order to ease problems of heat dissipation and X-ray intensity. An electron gun has not yet been designed, but the accompanying diagram suggests a general arrangement of electrodes at the output end of the helix.

It is further suggested that the spreading of the electron beam under its own space-charge forces may be prevented by applying an axial magnetic field along the guide by means of a long solenoid. The electrons then acquire a cycloidal component of motion in a plane normal to the axis, similar to the motion in a cylindrical magnetron. Assuming the initial radius of the beam to equal that of the proton beam (1 $\mathrm{mm}$.$) , and using the magnetron$ cut off equation, one can calculate the magnetic field required to prevent the beam radius from exceeding the value needed to keep the proton beam properly focused. Values obtained in this way range from 870 gauss at the input end of the accelerator to 570 gauss at the output end. The divergent field at the open end of the solenoid may be used, with careful design, to guide the electrons from the annular cathode in towards the axis.

The spread of energy in the emerging proton beam will depend on how far the stable phase-point is in advance of the peak of the field. Taking this phase angle to be $35^{\circ}$, the maximum amplitude of phase excursion a particle can have is $70^{\circ}$. The maximum kinetic energy such a particle can have relative to the wave is that due to this phase excursion through the axial field, and amounts to $50 \mathrm{keV}$. at the output end. Converting this to velocity, transforming to the laboratory frame of reference, and reconverting to energy, one finds that the energy spectrum of the output beam has a maximum value of \pm 10 per cent, 